\title{
CRISPR technology-a brave new world for microbes
}

\section{Editorial}

Microbes provide a basic segway for the continuum of genetic discovery and manipulation. They serve as a basis for discovery due to the ease of experimentation and the array of assay development that can be accomplished in a short amount of time with little expense. The recent development of a CRISPR/Cas9 system to cut or deliver genetic material has been developed using bacteria. The implications for cutting out portions of

DNA and replacing it with new DNA lie, of course, in the transference of this ability from bacterial model systems to the human genome and the possibilities of cutting out and replacing those pieces of the human genome that carry illness and disease. As can be anticipated, ethical issues abound as one contemplates moving in this direction. We give tribute to those who were the first to discover ways to transfer genetic material, but also greatly acknowledge all who have contributed to the efforts in genetic engineering of bacteria through the years, both preceding and following all major discoveries. Herbert Boyer and Stanley Cohen first accomplished genetic engineering as the direct transfer of DNA from one organism to another in 1972. Development work in this area led through the years to the first description of what would later be called CRISPR. It came from researcher Yoshizumi Ishino and his colleagues in 1987 at Osaka University. They accidentally cloned part of a CRISPR gene region together with the iap gene, their target of interest. Then most recently, the U.S. Patent and Trademark Office decided to grant two new CRISPR patents to UC Berkeley, home of Jennifer Doudna, a biochemist who many consider the creator of CRISPR, giving her legal ownership of the unique invention.

CRISPR is an abbreviation for Clustered Regularly Interspaced Short Palindromic Repeats and is a family of DNA sequences naturally found within the genomic content of bacteria. How is CRISPR different from conventional gene therapy? Removing or editing a gene using conventional gene therapy is imprecise. However, with CRISPR/Cas9, scientists are gaining the ability to reconstruct the genome at will by correcting bits of DNA in the genome, some as small as a single DNA base pair. The term CRISPR/Cas9 stands for CRISPR plus the associated protein Cas9. When a bacteriophage virus that has previously infected a bacterium attacks it again, an enzyme called a CRISPR associated protein (Cas protein) finds and cuts the viral DNA. This eliminates the infection and manifests as an inherent immune mechanism for the bacterium. Another aspect of the CRISPR technology that makes the system work involves the presence of the PAM or Protospacer Adjacent Motif. This is a 2-6 base pair DNA sequence immediately following the sequence targeted by the Cas9 nuclease. PAM is a component of the invading virus, but is not a component of the bacterial CRISPR locus. It is required however for the bacterial Cas9 nuclease to be able to cut the viral DNA. One of the latest research tools is the genome editing RNA-guided CRISPR Cas9 system that permits highly specific genomic modifications. Guide RNA (gRNA) or shortguide RNA (sgRNA) is an important component of the CRISPR Cas9 system. Its importance extends to the ability to target specific areas of the DNA. In order to accomplish this, the design of the gRNA sequence becomes critical.
Volume 6 Issue 6 - 2018

\author{
Sheila Wood \\ Rowan University, USA
}

Correspondence: Sheila Wood, Rowan University, Glassboro, NJ, USA, Email sjwood@twc.com

Received: December 18, 2018 | Published: December 27, 2018

Gene editing has given scientists the ability to change a bacterium's DNA by harnessing this naturally occurring genome editing system in bacteria. The bacteria capture parts of DNA from invading viruses and create DNA segments, or CRISPR arrays, from them. These arrays are used by the bacteria to recognize the viruses. When the virus invades again, the bacteria produce RNA from the created CRISPR arrays to target the viral DNA. Bacteria then use the Cas9 enzyme to cut the DNA apart and disable the virus. Using this technology in the lab, researchers in mammalian genetics create a small piece of RNA with a guide sequence that attaches to a target sequence of DNA. This RNA also binds to the Cas9 enzyme. As in bacteria, the modified RNA is used to recognize the DNA sequence, and the Cas9 enzyme cuts the DNA at the targeted location. Once the DNA is cut, researchers use the cell's own DNA repair machinery to add pieces of genetic material. They can also make changes to the DNA by replacing an existing segment with a customized DNA sequence. As scientists become more astutely aware of the possibilities for helping prevent complex diseases such as cystic fibrosis and sickle cell disease, the question becomes how and when do such studies move from animal models to humans. Although genome editing has great potential in the prevention and treatment of disease, research is done using cells and animal models. Scientists must determine safe and effective approaches for use in humans. Could such diseases as heart disease, mental illness, or cancer and AIDS benefit from this technology? ${ }^{1-4}$

Ethical concerns become paramount when germline DNA is changed in order to treat or eliminate disease conditions. When certain human genomes are altered, it affects what passes from generation to generation. Without highly controlled knowledge of what may happen future generations from the change, how can such choices be confidently made? Based on concerns about safety, germline and embryo gene editing are illegal in some countries. On the other hand, what greater relief for families to know that their descendants will not have to suffer such conditions as mental illness, alcoholism, breast cancer, heart disease, cystic fibrosis, epilepsy, and countless other conditions that limit the quality of life or even the ability to live an independent life. Decisions that center on less impactful treatments include those changes that are limited to cells other than egg and sperm and affect only certain tissues. These are not passed from one generation to the next. Such changes are not as ethically challenged as those that will affect future generations. So, it seems this case brings the gift of scientific potential along with the grave responsibility for clear and ethical approaches. The capability for treating families across generations becomes more of a possibility with each successive experiment. How do we prepare, how do we set goals, how do we 
apply controls, how do we develop standards for experimentation, how do we manifest our growing knowledge, what are the limits? These questions I leave with you. Handle them well.

\section{Acknowledgments}

None.

\section{Conflicts of interest}

Author declares that there is no conflict of interest.

\section{References}

1. Gupta RM, Musunuru K. Expanding the genetic editing tool kit: ZFNs, TALENs, and CRISPR-Cas9. J Clin Invest. 2014;124(10):4154-4161.

2. Hsu PD, Lander ES, Zhang F. Development and applications of CRISPRCas9 for genome engineering. Cell. 2014;157(6):1262-1278.

3. Komor AC, Badran AH, Liu DR. CRISPR-Based Technologies for the Manipulation of Eukaryotic Genomes. Cell. 2017;168(1-2):20-36.

4. Lander ES. The Heroes of CRISPR. Cell. 2016;164(1-2):18-28. 